\title{
HLA- A \& B Association in Psoriasis in Tamil Speaking South Indian Population
}

\author{
Ashwin Anandan ${ }^{1}$, Ravindraprasad Thokala ${ }^{2}$, Krishnamoorthy \\ Radhakrishnan ${ }^{3}$, Vinod Kumar Panicker ${ }^{4}$, Murugan Sundaram ${ }^{5}$
}

\begin{abstract}
${ }^{1}$ Department of Transfusion Medicine, Sri Ramachandra Medical College and Research Institute, Chennai, Tamilnadu, India. ${ }^{2}$ Department of Transfusion Medicine, Sri Ramachandra Medical College and Research Institute, Chennai, Tamilnadu, India. ${ }^{3}$ Department of Transfusion Medicine, Sri Ramachandra Medical College and Research Institute, Chennai, Tamilnadu, India. ${ }^{4}$ Department of Transfusion Medicine, Sri Ramachandra Medical College and Research Institute, Chennai, Tamilnadu, India. ${ }^{5}$ Department of Dermatology, Sri Ramachandra Medical College and Research Institute, Chennai, Tamilnadu, India.
\end{abstract}

\section{ABSTRACT}

\section{BACKGROUND}

Psoriasis is an auto-immune skin disease affecting skin, nails and joints. The association of HLA with psoriasis is already established with HLA- $\mathrm{C}^{*} 06$ known to be associated strongly with the disease. We wanted to determine the HLA -A \& HLA-B pattern and its association with psoriasis in a Tamil speaking ethnic population.

\section{METHODS}

A total of 100 psoriasis patients attending the Dermatology OPD at SRMC were taken up for the study. This was a case control study and hence 100 voluntary blood donors donating at the SRMC Hospital blood bank were taken up for study as controls. Voluntary blood donors are considered as healthy normal individuals and hence chosen as controls. All the 100 patients and 100 controls were typed for HLA (Human Leucocyte Antigen) - A \& B by PCR-SSP (Polymerase Chain Reaction-Sequence Specific Primers) and the results were analysed statistically using OpenEpi software ( 2 X 2 table). The Odds Ratio (OR), p (probability) value, and 95\% confidence interval were the statistical tests which were studied.

\section{RESULTS}

HLA-A*02, 24 and HLA-B*35 were found to be strongly associated with psoriasis among Tamil speaking ethnic population.

\section{CONCLUSIONS}

There are different HLA - A \& B alleles associated with psoriasis in Tamil ethnic population in comparison with other ethnic studies.

\section{KEY WORDS}

Human Leucocyte Antigen, Polymerase Chain Reaction-Sequence Specific Primers, psoriasis, Odds Ratio, Alleles, Tamil Ethnicity
Corresponding Author:

Dr. Ravindraprasad Thokala,

Department of Transfusion Medicine,

Sri Ramachandra Medical College and Research Institute, Porur, Chennai-600116,

Tamilnadu, India.

E-mail: drravi1212@gmail.com

DOI: $10.14260 /$ jemds/2020/390

Financial or Other Competing Interests: None.

How to Cite This Article:

Anandan A, Thokala R, Radhakrishnan K, et al. HLA- $A$ \& $B$ association in psoriasis in tamil speaking South Indian population. J. Evolution Med. Dent. Sci. 2020;9(23): 17821786, DOI: 10.14260/jemds/2020/390

Submission 18-02-2020,

Peer Review 25-03-2020,

Acceptance 27-03-2020,

Published 08-06-2020.

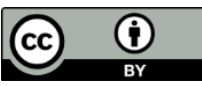




\section{BACKGROUND}

Robert Williams first described psoriasis. The word psoriasis was derived from 'PSORA' a Greek word in the $19^{\text {th }}$ century meaning scale.[1] Psoriasis is an inflammatory skin disease which is quite resistant to treatment. Psoriasis affects $1-3 \%$ of the population worldwide.[2] There is a clear genetic role of HLA, T-cells and APC (Antigen Presenting Cells) in the pathogenesis of the disease.[3] The major clinical feature of psoriasis is localized chronic inflammation and scaly erythematous plaques which is sometimes painful and sometimes pruritic. Psoriasis lesions consist of red scaly sharply demarcated indurated plaques seen on the extensor surfaces and the scalp. This disease is usually associated with variation in duration and extent with periods of flare and remission. ${ }^{[4,5]}$ The overall incidence was 57.3 per 1 lakh population, for men 54.4 per 1 lakh population, and for women 60.2 per 1 lakh population.[6] Epidemiological studies have estimated the worldwide prevalence of psoriasis to be between $0.6 \%$ - 4.8\%.[7] Prevalence studies from India are mostly hospital based and hence therefore the data may not be representative of the general population. ${ }^{[8]}$

There are various clinical types of psoriasis. It is broadly classified into Pustular and Non-Pustular psoriasis. Among the different types, Chronic Plaque type psoriasis behaves differently in different individuals. Appearance of Chronic Plaque psoriasis consist of a typical lesion which is characteristic and may be single or usually multiple, well defined erythematous plaques with silvery white scales and a sharply delineated edge. Guttate Type psoriasis demonstrates the acute onset of a myriad of small $2-10 \mathrm{~mm}$ diameter scaly lesion of psoriasis. It usually occurs shortly after an acute streptococcal infection of the tonsils: usually seen in children and occasionally in adults. Flexural inverse psoriasis affects the infra-mammary, perineal and axillary regions. It appears as red shiny well demarcated plaques. It can mimic fungal infections and underlying immune deficiency must be ruled out. Palmoplantar psoriasis affects the palms and soles which may present as typical scaly patches on which a fine silvery scale can be seen.

Erythrodermatic psoriasis patients have total or sub-total involvement of the skin. There is thermo regulation compromise leading to hypothermia and protein loss in scales. Pustular psoriasis can be generalized or localized to the palms and soles. These patients require hospital admission for management. The patient is pyrexial, with red painful and inflamed skin with monomorphic sterile pustules which may coalesce to form sheets. Psoriatic Arthropathy (PsA) is defined as a distinct entity under seronegative spondyloarthropathies and is given simple criteria for its diagnosis which includes an inflammatory arthritis, presence of psoriasis and absence of RA factor. ${ }^{[9]}$

\section{Pathogenesis}

The hallmark of psoriasis is sustained inflammation leading to hyper proliferation of keratinocytes and dysfunctional differentiation. The histologic findings are acanthosis (epidermal hyperplasia). Neovascularisation is also a prominent feature. There are disturbances in the innate and adaptive cutaneous immune responses which is responsible for the development of the psoriatic inflammation. There is an activation of the innate immune system which is driven by the endogenous danger signals and cytokines which characteristically co-exists with an auto inflammatory perpetuation in some patients and $\mathrm{T}$-cell driven auto immune reactions in others. Hence psoriasis has been sharing trades of an autoimmune disease with these both mechanisms overlapping and even potentially with one another.[10,11] The development of psoriatic plaque is not restricted to inflammation in the epidermal layer, but rather is shaped by the interaction of the keratinocytes with different cell types (innate and adaptive immune cells and vasculature) spanning the dermal layer of the skin. The pathogenesis of psoriasis can be conceptualized into a initiation phase possibly triggered by trauma (Koebner phenomenon), infection, drugs and a maintenance phase characterized by a chronic clinical progression.[12]

\section{HLA and psoriasis}

Genome wide linkage studies of psoriasis affected families have so far detected at least 60 chromosomal loci linked to Psoriatic susceptibility, the most prominent locus is PSORS-1 which has been attributed in up to $50 \%$ of the patients with the disease.[13] PSORS-1 is located on chromosome 6p21 within the major histocompatibility complex (MHC) which is specifically in the class-I telomeric region of HLA-B. HLA-Cw6 is strongly linked to early and acute onset psoriasis.[14] HLA association with psoriasis has been well established with HLA$C^{*} 06$ showing strong association with the disease. But studies showing HLA-A and HLA-B association with psoriasis from this ethnicity are few..[4] Only one study done on 50 samples from south Indian population showed that HLA-B*18, HLA$B * 35$ and HLA-B*40 had a strong association with psoriasis.[15] There is a lacunae in determining the HLA association with psoriasis Hence this study was undertaken to determine the HLA-A \& HLA-B association with psoriasis. This study was undertaken to determine the HLA-A \& HLA-B pattern and its association with psoriasis among the Tamil speaking ethnic population.

\section{METHODS}

This was a case control study, conducted by the Department of Transfusion Medicine, Dermatology and Human Genetics at a tertiary care teaching hospital. The study was undertaken from April 2014 to March 2017. A total of 100 psoriasis patients who attended the Dermatology OP and 100 normal healthy controls who were voluntary blood donors donating at SRMC blood bank were taken up for the study. Psoriasis prevalence at SRMC is around $2 \%$ and the annual patients attending dermatology OPD is around 5000. Hence the sample size was arrived at 100 since $2 \%$ of 5000 is 100 . All newly diagnosed psoriasis cases and psoriasis patients on treatment were included in the study. PASI (psoriasis Area Severity Index) score was calculated by the Dermatologist at the Dermatology OPD during the first visit. Blood samples for HLA typing were collected after obtaining informed consent from the participants. HLA typing for Class I - A \& B was done by PCR-SSP method in the Department of Human Genetics. Institutional Ethics Committee approval (Ref: IECN1/11/OCT/25/62) had been obtained for the study.

Blood samples $(3 \mathrm{ml})$ were collected in sterile EDTA containers from 100 psoriasis patients and 100 healthy blood 
donors for HLA typing. DNA was isolated from the peripheral blood by Miller's method. DNA isolation was performed using Qualigen diagnostics as per the kit inserts provided and SOP followed. Isolated DNA was stored in $-70^{*} \mathrm{c}$ freezer in the Department of Human Genetics for HLA typing to be done. HLA typing was performed using BAG health care $\mathrm{GmbH}$ kits (Combo kits for HLA A \& B for 100 cases and controls). SOP provided by Department of Human Genetics was followed regarding setting up of PCR thermal cycler, master mix preparation and agarose gel electrophoresis. HLA -A and HLA$B$ results were interpreted.

\section{RBC Lysis}

To the peripheral blood $40 \mu \mathrm{l}$ of RBC lysis buffer was added mixed thoroughly and incubated at room temperature for 1 hour. Samples were then centrifuged at $2000 \mathrm{rpm}$ for $20 \mathrm{~min}$.

\section{DNA Precipitation}

The resulting supernatant was transferred to a centrifuge tube and $7 \mathrm{ml}$ of isopropyl alcohol was added. The tube was mixed gently until the DNA precipitated. The precipitated DNA was then transferred to a $1.5 \mathrm{ml}$ sterile micro centrifuge tube and $500 \mu$ litre of $70 \%$ ethanol was added and spun at $2000 \mathrm{rpm}$ for $5 \mathrm{~min}$. at $4{ }^{\circ} \mathrm{C}$. Ethanol was discarded, DNA was air dried and then dissolved in $150 \mu \mathrm{l}$ of buffer. The sample was then refrigerated at $4{ }^{\circ} \mathrm{C}$ for complete dissolution and stored at $20{ }^{\circ} \mathrm{C}$ till further use.

\section{Spectrophotometric Analysis}

The quality and quantity of the DNA sample was assessed by spectrophotometer. $10 \mu \mathrm{l}$ of the DNA was diluted with $990 \mu \mathrm{l}$ of TE buffer and OD (Optical Density) was read at a wavelength of $260 \mathrm{~nm}$ and $280 \mathrm{~nm}$. The concentration of the samples was determined based on the OD ratio. 10D corresponds to 50 $\mathrm{ng} / \mathrm{ml}$ of double stranded DNA. Samples with $260 \mathrm{~nm}$ and 280 nm OD ratio between 1.7 to 1.9 were selected for PCR.

\section{PCR (Polymerase Chain Reaction)}

Amplification of the gene was performed using specific primers under appropriate cycling conditions of denaturation, annealing and extension in a thermal cycler.

\begin{tabular}{|c|c|c|c|c|}
\hline Sl. No. & \multicolumn{2}{|c|}{ Reagents } & $\begin{array}{c}\text { Working } \\
\text { Concentration }\end{array}$ & $\begin{array}{c}\text { Working Volume } \\
(\mu \mathrm{l})\end{array}$ \\
\hline 1 & \multicolumn{2}{|c|}{$\begin{array}{l}\text { Taq DNA Polymerase } \\
\text { buffer }\end{array}$} & $1 \mathrm{x}$ & 2 \\
\hline 2 & \multicolumn{2}{|c|}{ dNTP mix } & $200 \mu \mathrm{l}$ & 0.4 \\
\hline 3 & \multicolumn{2}{|c|}{ Primer forward } & $50 \mathrm{pm}$ & 0.5 \\
\hline 4 & \multicolumn{2}{|c|}{ Primer reverse } & $50 \mathrm{p}$ & 0.5 \\
\hline 5 & \multicolumn{2}{|c|}{ Taq DNA polymerase } & 1.5 & 0.5 \\
\hline 6 & \multicolumn{2}{|c|}{ Water (Nuclease free) } & - & 15.1 \\
\hline 7 & \multicolumn{2}{|c|}{ Template } & $100 \mathrm{~nm}$ & 1 \\
\hline & & & Total $=$ & $20 \mu \mathrm{l}$ \\
\hline \multicolumn{5}{|c|}{ PCR Mix Preparation } \\
\hline \multicolumn{2}{|c|}{ Steps } & TemI & ature (in $\left.{ }^{\circ} \mathrm{C}\right)$ & Time \\
\hline \multirow{2}{*}{\multicolumn{2}{|c|}{$\begin{array}{l}\text { Initial denaturation } \\
\text { Denaturation }\end{array}$}} & & 94 & $5 \mathrm{~min}$ \\
\hline & & & 94 & $45 \mathrm{sec}$ \\
\hline \multicolumn{2}{|c|}{ Annealing } & ${ }^{*}$ Differe & or different genes & ${ }^{*}$ Different for different genes \\
\hline \multirow{2}{*}{\multicolumn{2}{|c|}{ Extension }} & & 72 & $45 \mathrm{sec}$. to $1 \mathrm{~min}$ \\
\hline & & \multicolumn{3}{|c|}{ Repeated for 30 cycles } \\
\hline \multicolumn{2}{|c|}{ Final extension } & & 72 & $5 \mathrm{~min}$ \\
\hline \multicolumn{5}{|c|}{ PCR Conditions } \\
\hline
\end{tabular}

A master mix comprising of all components except the template DNA was prepared and aliquoted in separate tubes. The template DNA was then added, the tubes were placed in the thermal cycler and subjected to the standardized PCR conditions. The PCR conditions were standardized for each gene.

\section{PCR Confirmation}

PCR amplification was confirmed by $2 \%$ agarose gel electrophoresis. 100 bp DNA molecular weight marker was used to confirm the amplicon size.

\section{Agarose Gel Electrophoresis}

Analysis of PCR product was done by a $2.4 \%$ agarose gel electrophoresis. The gel was electrophoresed at $4 \mathrm{~V} / \mathrm{cm}$ and visualized in the gel documentation system.[16]

\section{PASI (psoriasis Area and Severity Index) Score}

This was developed by Fredricksson and Petterson in 1978. Both intensity and extent of the Psoriatic plates are calculated separately for four anatomical regions (head, trunk, upper and lower extremities) by the dermatologist. The intensity of erythema, desquamation and induration is rated on a 5 point scale with 0 - no involvement, 1- slight, 2-Moderate, 3-Severe, 4-Very Severe characteristics. The percentage of involvement of the four anatomical regions is assigned a numerical value of 0-6 with 0 - no involvement, $1-1$ to $9 \%, 2-10$ to $29 \%, 3-30$ to $49 \%, 4-50$ to $69 \%, 5-70$ to $89 \%, 6-90$ to $100 \%$ of body surface area involvement (BSA). When calculating the PASI score four anatomical regions are calculated according to the proportion of the whole involvement of the skin. The PASI score varies from 0 to 72 . Higher score indicates severe conditions. The main advantage of PASI score is that it is the gold standard in clinical trials.[17]

\section{Statistical Analysis}

The Odds ratio (OR), $\mathrm{p}$ (probability) value, and 95\% confidence interval were the statistical tests which were studied using open epidemiology software version 3.01. This is free and open software for epidemiological statistics. Licenses are available in full text at opensource.org/license. This study was a case control study. $\mathrm{p}$ Value of $<0.5$ was considered statistically significant. Any HLA allele having a OR of $>1$ and with a $p$ value of $<0.5$ was considered to have a strong positive association with psoriasis and similarly any HLA allele having a OR $<1$ and with a $\mathrm{p}$ value of $<0.5$ was considered to have a negative association (may be protective against the disease).

\section{RESULTS}

As an individual allele, HLA-A*02, HLA-A*11, HLA-A*24 and HLA-A*33 are the alleles to be found more in the cases implying their strong association with the disease as shown in Table 1. All these four alleles was predominantly found to be in male patients, late onset psoriasis and in patients with moderate PASI score (5-10). HLA-A*33 had the highest OR of 4.235. The above results imply that these alleles are strongly associated with psoriasis. HLA-A*01 was found in higher frequency among the controls. It was found in 35 controls of the 100 normal healthy controls (35\%) with Odds of 0.2532 with p-value of 0.0012 implying HLA-A*01 has a negative association with psoriasis (may be having a protective effect). 


\begin{tabular}{|c|c|c|c|c|c|c|c|c|c|c|c|c|}
\hline 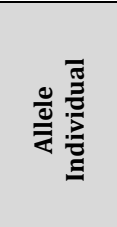 & 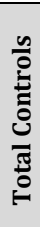 & 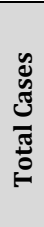 & 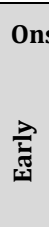 & 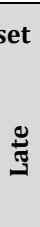 & $\stackrel{\frac{0}{\pi}}{\sum}$ & 苞 & $\begin{array}{r}\text { Se } \\
\text { (I }\end{array}$ & $\begin{array}{l}\stackrel{0}{a} \\
\stackrel{0}{2} \\
\text { n }\end{array}$ & $\begin{array}{l}\text { ty } \\
\text { l) }\end{array}$ & ڤี & 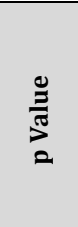 & 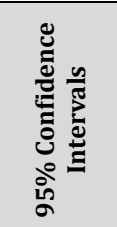 \\
\hline HLA-A*2 & 26 & 45 & 13 & 32 & 33 & 12 & 13 & 21 & 11 & 2.329 & 0.0049 & $1.284-4.225$ \\
\hline HLA-A*11 & 16 & 34 & 8 & 26 & 28 & 6 & 8 & 19 & 7 & 2.705 & 0.0032 & $1.376-5.317$ \\
\hline HLA-A*24 & 20 & 35 & 14 & 21 & 28 & 7 & 13 & 19 & 3 & 2.154 & 0.0175 & $1.136-4.082$ \\
\hline HLA-A*33 & 4 & 15 & 6 & 9 & 14 & 1 & 5 & 8 & 2 & 4.235 & 0.0079 & $1.353-13.25$ \\
\hline HLA-A*01 & 35 & 12 & \multicolumn{7}{|c|}{ Not applicable } & 0.2532 & 0.0012 & $\begin{array}{c}0.1221- \\
0.5254\end{array}$ \\
\hline \multicolumn{13}{|c|}{$\begin{array}{l}\text { Table 1. Individual HLA- A Allele Results } \\
\text { (Statistically Significant Findings) }\end{array}$} \\
\hline
\end{tabular}

\begin{tabular}{|c|c|c|c|c|c|c|c|c|c|c|c|}
\hline 冚 & 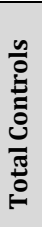 & 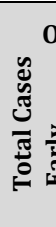 & 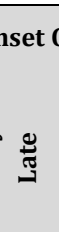 & $\stackrel{0}{\sum}$ & 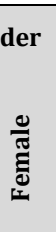 & $\begin{array}{r}\text { Sev } \\
(P\end{array}$ & $\begin{array}{l}\text { 욱 } \\
1 \\
\text { 1 }\end{array}$ & $\stackrel{ }{\wedge}$ & ฮ̆ & 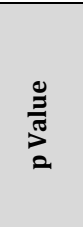 & 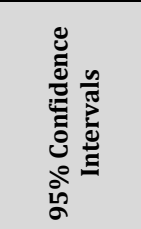 \\
\hline HLA-B* 18 & 4 & 154 & 11 & 10 & 5 & 3 & 10 & 2 & 4.23 & 79 & 1.35 \\
\hline 27 & 1 & 144 & 10 & 12 & 2 & 4 & 5 & 5 & & & 2.0 \\
\hline HLA-B* 35 & 19 & 3610 & 26 & 3 & 6 & 10 & 19 & 7 & 23 & 70 & \\
\hline HLA-B* 40 & 10 & 246 & 18 & 17 & 7 & 6 & 16 & 2 & 2.842 & 0.0084 & $1.279-6.315$ \\
\hline HLA-B* 13 & 14 & 3 & & No & applic & cable & & & 0.19 & 0.0052 & $\begin{array}{c}0.0528- \\
0.6835\end{array}$ \\
\hline & & & & & & & & & & & \\
\hline
\end{tabular}

\begin{tabular}{|c|c|c|c|c|c|c|c|c|c|c|}
\hline 造 & 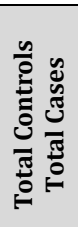 & 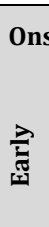 & 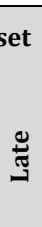 & $\frac{\varrho}{\Sigma}$ & 营 & $\begin{array}{c}\text { Sev } \\
\text { (P } \\
\text { in } \\
v\end{array}$ & $\begin{array}{c}0 \\
1 \\
1 \\
\text { in }\end{array}$ & 롯 & 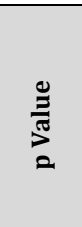 & 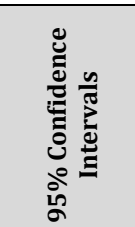 \\
\hline HLA-A*2,24 & 212 & 6 & 6 & 9 & 3 & 6 & 42 & 26.682 & 0.0055 & $1.455-30.68$ \\
\hline HLA- B* 15,35 & 210 & 3 & 7 & 7 & 3 & 3 & 52 & 25.444 & 0.0172 & $1.162-25.52$ \\
\hline
\end{tabular}

The four HLA-B alleles that were found in higher frequency among the patients were HLA-B*18, HLA-B*27 and HLA-B*35 and HLA-B*40 as shown in Table 2 . HLA-B*35 was the highest allele (as an individual allele) to be found in highest number of patients i.e $36 \%$ with an OR 2.398. As an allelic combination, HLA-A* 2, 24 and HLA-B*15, 35 was found in 12 and 10 patients respectively. Among the HLA-B alleles, these 4 alleles as shown in Table 2 was found predominantly among male patients, late onset psoriasis and in patients with moderate PASI score (5-10). HLA-B*27 was found in 14 patients with the highest OR of 16.12 . HLA-B*13 had a negative association with psoriasis as it was found in 14 controls, with OR 0.19 and pvalue of 0.0052 implying it may have a protective effect against the disease.

\section{DISCUSSION}

This study was carried out to identify the HLA alleles associated with psoriasis and comparing with normal healthy population. HLA- $\mathrm{C}^{*} 6$ has been proven to be strongly associated with psoriasis from various ethnic population worldwide. ${ }^{[4]}$ Comparing these findings with the current study, there are certain other HLA alleles also to be considered having association with psoriasis. HLA- $A^{*} 2$, HLA- $A^{*} 11$, HLA$A^{*} 24$, HLA- $B^{*} 18$, HLA- $B^{*} 27$ and HLA- $B^{*} 35$ are some of the alleles to have a positive association with psoriasis. HLA- B*27 in particular was found associated in patients with Psoriatic Arthropathy.[14] This finding of HLA- B*27 is concordant with other studies.

\section{HLA Association in Different Ethnic Populations}

HLA- $A^{*} 1$, HLA- B*37 was found to have a strong association with psoriasis from a Korean ethnic population.[13] Similar study from Turkey showed HLA- ${ }^{*} 30$ and HLA- B*50 to have a strong association with psoriasis.[18] A study from Japan showed HLA- A*1, HLA- A*2, HLA- B*13, and HLA- B*39 to be associated with the disease.[19] Studies from within India have shown different HLA being associated with psoriasis. ${ }^{[8]}$ HLA$B^{*} 13$ was seen associated with Psoriatic patients from north India ethnic population. Only one study from South India has shown HLA- B*57 to be associated with psoriasis.

There are various studies showing HLA-C*06, being strongly associated with psoriasis.[13] Russel et al first reported in 1972, that HLA-B*13 and HLA-B*17 to be associated with psoriasis ${ }^{[20]}$ Chablani et al showed HLA-B*17 association with psoriasis. ${ }^{[21]}$ A study from North India showed HLA-A*01 and HLA-B*17 in increased frequency among the patients. ${ }^{[22]}$ Studies from India has shown different HLA associated with psoriasis. Comparing these results from previous studies, this study shows different HLA alleles to be strongly associated with psoriasis.[8],[14] Our study showed HLA-A*02, HLA-A*11, HLA-A*24, and HLA-B*18, HLA-B*27, HLA-B*35 as the significant alleles associated with psoriasis. HLA-A*01 and HLA-A*13 were found in increased frequency among the controls implying these two alleles may be protective against the disease.

\section{CONCLUSIONS}

HLA association with psoriasis varies among different ethnic populations. HLA-A*02, HLA-A*11, HLA-A*24 and HLA-B*18, HLA-B*27, HLA-B*35 have positive association with psoriasis whereas HLA-A*01 and HLA-B*13 have a negative association with psoriasis in the Tamil speaking ethnic population. Identifying the HLA associated with psoriasis helps the clinician in better understanding of the disease pathogenesis and management by discovery of newer generation biologicals.

Limitations

This study was done on a small sample size of 100 cases and 100 controls, because of the high cost involved.

\section{REFERENCES}

[1] Christopher E, Mrowietz U. Psoriasis. Braun-Falco's Dermatology. Vol. 1. $3^{\text {rd }}$ edn. Heidelberg: Springer 2010: p. 506.

[2] Neimann AL, Porter SB, Gelfand JM. The epidemiology of psoriasis. Expert Rev Dermatol 2006; 1:63-75.

[3] Richardson SK, Gelfand JM. Update on the natural history and systemic treatment of psoriasis. Adv Dermatol 2008; 24:171-96. 
[4] Indhumathi S, Rajappa M, Chandrashekar L, et al. The HLA C*6 allele as a possible genetic predisposing factor to psoriasis in South Indian Tamils. Archives of Dermatological Research 2016; 308(3):193-9.

[5] Griffiths CE, Barker JN. Pathogenesis and clinical features of psoriasis. Lancet 2007; 370(9583):263-71.

[6] Bell LM, Sedlack R, Beard CM, et al. Incidence of psoriasis in Rochester, Minn, 1980-1983. Arch Dermatol 1991; 127(8):1184-7.

[7] Gelfand JM, Weinstein R, Porter SB, et al. Prevalence and treatment of psoriasis in the United Kingdom: a population based study. Archives Dermatol 2005; 141(12):1537-41.

[8] Dogra S, Yadav S. Psoriasis in India: Prevalence and pattern. Ind J Dermatol Venerol Leprol 2010; 76(6):595601.

[9] Di Megilo P, Villanova F, Nestle FO. Psoriasis. Cold Spring Harb Perspect Med 2014; 4(8):a015354.

[10] Harden JL, Krueger JG, Bowcock AM. The immunogenetics of psoriasis- a comprehensive review. J Autoimun 2015; 64:66-73.

[11] Liang Y, Sarkar MK, Tsoi LC, et al. Psoriasis: a mixed autoimmune an autoinflammatory disease. Curr Opin Immunol 2017; 49:1-8.

[12] Bowcock AM, Krueger JG. Getting under the skin: the immunogenetics of the psoriasis. Nat Rev Immunol 2005; 5(9):699-711.
[13] Nair RP, Stuart PE, Nistor I, et.al. Sequence and haplotype analysis supports HLA-C as the psoriasis susceptibility 1 gene. Am J Hum Genet 2006; 78(5):827-51.

[14] Shankarkumar U. HLA-C allele association in Mumbai psoriasis patients. Int J Hum Genet 2012:12(1):41-4.

[15] Anandan A, Radhakrishnan K, Thokala RP, et al. HLA-B association in psoriasis. International Journal of Medical Research and Review 2016:4(5):831-4.

[16] Miller SA, Dykes DD, Polesky HF. A simple setting out procedure for extracting DNA from human nucleated cells. Nucleic Acids Res 1998; 16(3):1215.

[17] Naldi L, Svensson A, Diepgen T, et al. Randomised clinical trials for psoriasis: 1977 to 2000: The Eden survey. J Invest Dermatol 2003; 120(5):738-41.

[18] Shankarkumar U, Ghosh K, Badakere S, et al. Novel HLA Class I alleles associated in Indian Leprosy Patients. J Biomed Biotech 2003 ; (3):208-11.

[19] Singh S, Singh U, Singh S. HLA in patients with psoriasis. Ind J Dermatol Venerol Leprol 2011; 77(4):535.

[20] Russel TJ, Schultes LM, Kuban DJ. HLA antigen associated with psoriasis. New Engl J Med 1972; 287:738-40.

[21] Chablani UA, Contractor NM, Gadgil RB. HLA and complement C4 studies in psoriasis Vulgaris. Natl Med J India 1992; 5(1):8-11.

[22] Rani R, Narayanan R, Fernandez MA, et al. Role of HLA B and $\mathrm{C}$ alleles in the development of psoriasis in patients from North India. Tissue Antigens 1998; 51(6):618-22. 